\title{
ORIGINAL
}

\section{EXCESOS DE MORTALIDAD EN ESPAÑA DURANTE LA TRANSMISIÓN DE GRIPE PANDÉMICA EN EL AÑO 2009}

\section{Imaculada León Gómez (1),Víctor Manuel Flores Segovia (1), Silvia Jiménez Jorge (1, 2), Amparo Larrauri Cámara $(1,2)$, Rocío Palmera Suárez $(1,2)$ y Fernando Simón Soria $(1,2)$}

(1) Centro Nacional de Epidemiología - Instituto de Salud Carlos III.

(2) CIBER de Epidemiología y Salud Pública (CIBERESP).

\section{RESUMEN}

Fundamento: El sistema español de monitorización de la mortalidad y el «Programa Europeo de monitorización de excesos de mortalidad para la acción en salud pública» detectaron dos excesos de mortalidad en España en noviembre y diciembre de 2009. El objetivo de este trabajo es valorar su posible asociación con la transmisión de gripe pandémica.

Métodos: Se analizó la evolución de la mortalidad en España en los meses citados utilizando métodos de análisis de series temporales basados en las series históricas de mortalidad y se comparó en el tiempo con la transmisión de gripe.

Resultados: La mortalidad observada en la población total fue mayor de lo esperado en dos periodos: semanas 46-47/2009 con $5,75 \%$ de exceso y las semanas $51-52 / 2009$ con $7,35 \%$ de exceso. También se registró un exceso de mortalidad en niños de 5 a 14 años en las semanas 46-48/2009 con 41 defunciones vs las 21 esperadas. El exceso de mortalidad en noviembre fue concomitante con las mayores tasas de gripe. El exceso de diciembre se observó 5 semanas después del pico de gripe y coincidió con un descenso dramático de las temperaturas. El virus sincitial respiratorio y los accidentes de tráfico fueron descartados como factores asociados.

Conclusiones: Mientras que las temperaturas podrían explicar la mayoría del exceso de mortalidad observado en diciembre, ningún factor por si solo podría explicar el exceso de noviembre.

Palabras clave: Brote. Pandemia. Subtipo H1N1 del Virus de la Influenza A. Gripe humana. España. Mortality. Frío.

\section{ABSTRACT \\ Excess Mortality in Spain during transmission of pandemic influenza in 2009}

Background: The Spanish daily mortality monitoring system and the program «European monitoring of excess mortality for public health action» found two excesses of mortality in Spain in November and December 2009.

Methods: We analyzed the evolution of mortality in Spain during those months using time-series analysis methods based on historical mortality series and compared it in the time with influenza transmission.

Results: Observed mortality for the total population was higher than expected in two periods: weeks $46-47 / 2009$ with $5.75 \%$ excess and weeks $51-52 / 2009$ with $7.35 \%$ excess. Observed mortality higher than expected, was also observed in children 5-14 years old during weeks $46-48 / 2009$ with 41 deaths vs 21 expected. Exces mortality in November occurred before or was concomitant with highest influenza incidence rates. Excess mortality in December occurred five weeks after the influenza incidence peak and along with dramatic drop in temperatures. RSV and traffic accidents were ruled out as factor associated to these excesses.

Conclusions: While temperatures could explain most of the excess mortality observed in December, no single factor could be associated with observed excess mortality in November.

Key words: Epidemiology. Sentinel surveillance. Disease Outbreaks. Influenza A virus, H1N1 subtype. Influenza, human. Spain. Mortality. Cold Temperature.

Correspondencia:

Inmaculada León Gómez

Centro Nacional de Epidemiología

Instituto de Salud Carlos III.

C/ Sinesio Delgado 6.

28042 Madrid.

ileon@isciii.es

vflores@isciii.es 


\section{INTRODUCCIÓN}

La declaración de la pandemia por virus de la gripe A (H1N1) 2009 en Abril de 2009 motivó un cambio en la frecuencia de los análisis realizados por el sistema de monitorización de la mortalidad diaria (MoMo), de forma que se extendiera al periodo octubre-mayo la detección diaria de señales de alerta por excesos de mortalidad, en vez de la semanal como suele realizarse en este periodo. En Europa el programa «European monitoring of excess mortality for public health action» (Euro-MoMo) modificó la implementación de sus paquetes de trabajo, comenzando en septiembre de 2009 la fase prepiloto de modelos predictivos de mortalidad semanal con la participación de 6 países europeos, que se amplió a 13 países en la fase piloto que comenzó en octubre de 2009.

MoMo y Euro-MoMo son sistemas de detección precoz de señales de alerta asociadas a la mortalidad general. MoMo en España y Euro-MoMo en los 13 países que participan en la fase piloto detectaron señales de alerta por exceso de mortalidad en noviembre y diciembre de 2009.

En este artículo se presentan los resultados del estudio de las señales detectadas por MoMo y se valora su posible asociación con la transmisión de la gripe (H1N1) 2009.

\section{MATERIAL Y MÉTODOS}

Estudiamos la mortalidad en los meses de noviembre y diciembre de 2009 en los que se detectaron señales de alerta por mortalidad tanto en MoMo como en Euro-MoMo.

MoMo, desarrollado en el marco del «Plan de acciones preventivas contra los efectos de las temperaturas excesivas», desde 2004, durante el periodo junio-septiembre realiza un análisis diario de los datos de mortalidad de los municipios que cuentan con registros civiles informatizados (384 a 15 de enero del 2008). Estos municipios se distribuyen por todo el territorio e incluyen al $59 \%$ de la población ( $86 \%$ de los mayores de 74 años), según datos del padrón municipal a 1 de enero del $2007^{2}$.

En el año 2007 se inició el proyecto europeo Euro-MoMo, financiado por el Programa de Salud Pública de la Unión Europea, con la participación de 18 Estados miembros y en el que el Centro Nacional de Epidemiología es responsable de la evaluación de modelos predictivos. Los datos de MoMo, agregados semanalmente, son el componente español del Programa Euro-MoMo.

Para detectar y cuantificar los incrementos en mortalidad para el total de la población monitorizada en el periodo estudiado, MoMo utilizó como mortalidad esperada la estimada a partir de las series de mortalidad de los años 1999-2005 proporcionadas por el INE. Los años 2003 y 2006 se excluyeron de las series por los excesos de mortalidad observados, potencialmente asociados a olas de calor. La mortalidad esperada para cada día de 2009 se calculó mediante medias móviles de 5 días alrededor del día de interés de los 6 años incluidos en las series una vez eliminada la tendencia mediante regresión lineal y la variabilidad de los datos mediante transformación logarítmica. Se estimaron intervalos de confianza al $95 \%$ alrededor de la mortalidad diaria esperada. Esta se compara con la mortalidad diaria observada recibida diariamente de los registros civiles informatizados. Además, para identificar posibles periodos de incrementos significativos de la tendencia de la mortalidad, se realiza un análisis de tendencias a corto plazo mediante una adaptación del método CUSUM algorítmi$\mathrm{Co}^{3}$. Como umbral de alerta se utiliza el percentil 90 de la distribución de los valores de días consecutivos de los últimos dos años de la series históricas en los que la mortalidad observada supera la media esperada. 
En el análisis de mortalidad para grupos de edad con número de defunciones pequeño se utilizaron modelos lineales generalizados con link Poisson.

Los análisis con base semanal necesarios para comparar los resultados de MoMo, Euro-MoMo y el sistema de vigilancia de la gripe en España, utilizan la semana bajo norma ISO $8601^{4}$.

Los análisis realizados por MoMo se desarrollaron con el paquete estadístico R.

La información de la circulación de virus de la gripe (H1N1) 2009 se obtuvo del Sistema de Vigilancia de la Gripe en España $(\mathrm{SVGE})^{5}$. La tasa de detección viral para virus de la gripe se estima como el porcentaje semanal de muestras positivas a virus de la gripe en las diecisiete redes centinela integrantes del SVGE. Además se obtuvo el porcentaje de muestras positivas de virus respiratorio sincitial (VRS) de ocho laboratorios integrados en el SVGE que proporcionaron el denominador de las muestras semanales analizadas.

Se compararon la evolución y los excesos de mortalidad por todas las causas con la intensidad de la circulación de virus gripales y VRS en el periodo de estudio.

\section{RESULTADOS}

\section{Mortalidad en la población general}

En los meses de noviembre y diciembre de 2009 en el total de la población general se registraron 48.237 defunciones, que significaron el $2,78 \%$ más de las esperadas (46.453). La diferencia fue mayor en diciembre $(3,13 \%)$ que en noviembre $(2,38 \%)$ (tabla 1$)$.

En noviembre el análisis de tendencia mostró una mortalidad por encima de los promedios esperados durante 11 días consecutivos, 4 días por encima del umbral de alerta (figura 1). Este exceso correspondió a 8.574 defunciones observadas frente a 8.108 esperadas $(5,75 \%$ de exceso). El exceso medio diario en este periodo fue de 42,4 defunciones. Durante este periodo se observaron 2 días con mortalidad por encima del umbral de alerta para la mortalidad diaria (14 y 18 de noviembre) (figura 2).

En diciembre el análisis de tendencia muestra una mortalidad por encima de los promedios esperados durante 8 días consecutivos, 2 días por encima del umbral de alerta (figura 1). Este exceso corresponde a 7.034 defunciones observadas frente a 6.550

Tabla 1

Defunciones observadas, defunciones esperadas, porcentajes de variación y media de defunciones diarias para el total de los 384 municipios incluidos en MoMo en Noviembre y Diciembre de 2009

\begin{tabular}{|l|c|c|c|}
\hline \multicolumn{1}{|c|}{ Mortalidad } & Noviembre 2009 & Diciembre 2009 & Total \\
\hline Observada & 22.564 & 25.673 & 48.237 \\
\hline Esperada & 22.039 & 24.894 & 46.933 \\
\hline Diferencia (\%) & 2,38 & 3,13 & 2,78 \\
\hline Media defunciones diarias (SD) & $752,1(39,22)$ & $828,2(51,65)$ & $790,8(59,55)$ \\
\hline
\end{tabular}


Figura 1

Días consecutivos de mortalidad observada por encima de la media esperada para los 384 municipios informatizados 1 noviembre-31 diciembre 2009 ( $\longrightarrow$ ), umbral de alerta (- - - - - - - ), mediana de días consecutivos por encima de lo esperado dos años previos (

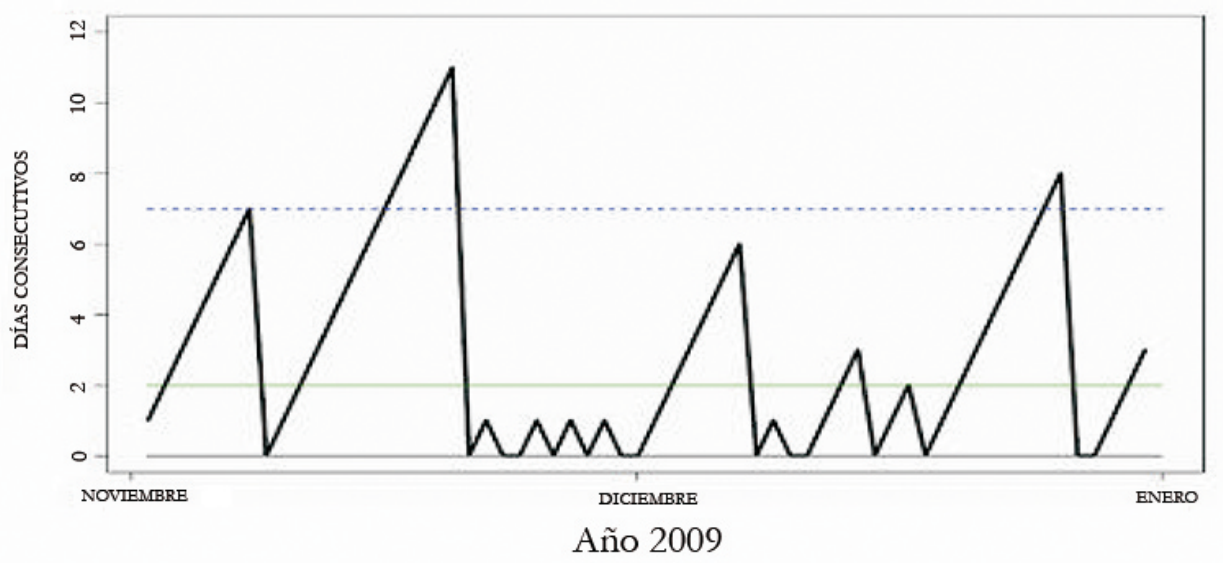

Figura 2

Defunciones diarias observadas en 384 municipios informatizados 1 noviembre-31 diciembre 2009 ( - ), defunciones esperadas (- - - - ) y umbral de mortalidad diaria 95\% ( - )

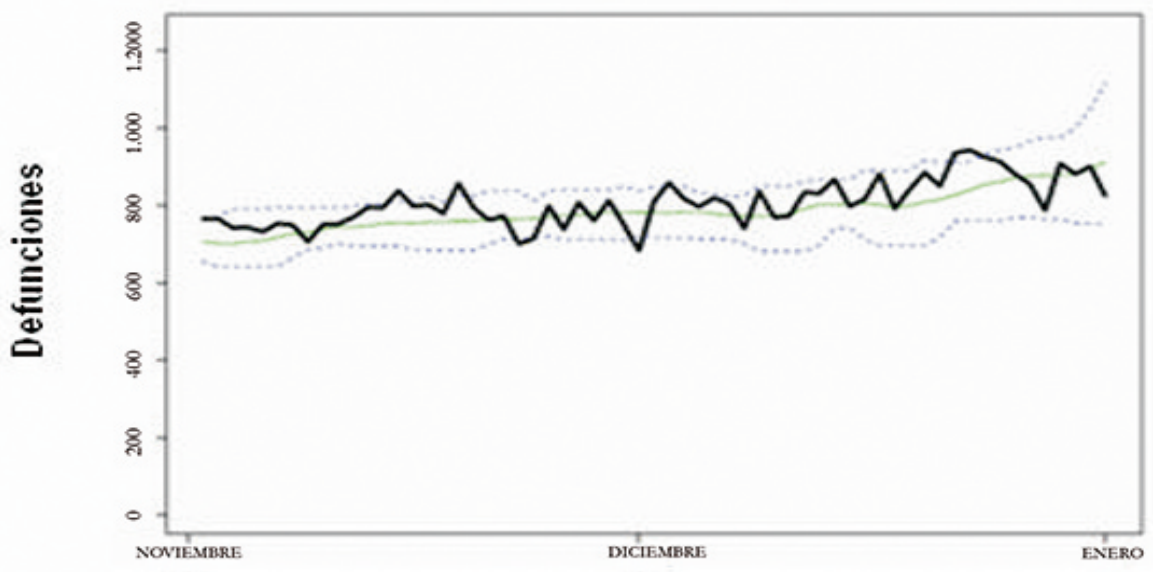

Año 2009

esperadas $(7,35 \%$ de exceso). El exceso medio diario en este periodo fue de 60,4 defunciones. Durante este periodo se obser- varon 2 días con mortalidad por encima del umbral de alerta para la mortalidad diaria (21 y 22 de diciembre). (Figura 2). 
Tabla 2

Defunciones observadas y esperadas en el grupo de edad de 5 a 14 años en los 384 municipios incluidos en MoMo, semanas 46-48 del 2009

\begin{tabular}{|c|c|c|c|c|}
\hline Semana & Observado & Esperado & $\begin{array}{c}\text { Límite superior } \\
\mathbf{9 5 \%}\end{array}$ & $\begin{array}{c}\text { Observado- } \\
\text { Esperado }\end{array}$ \\
\hline 46 & 15 & 6,7 & 7,47 & 8,3 \\
\hline 47 & 10 & 7,6 & 8,46 & 2,4 \\
\hline 48 & 16 & 7,06 & 7,82 & 8,94 \\
\hline Total & 41 & 19,6 & 23,75 & 21,4 \\
\hline
\end{tabular}

\section{Mortalidad por grupos de edad}

El análisis para los grupos de edad de personas mayores de 65 años siguió el mismo patrón observado en la mortalidad de la población general. En los grupos de menores de 65 años no se observó ningún exceso, a excepción del grupo de 5 a 14 años, en el que se identificó un exceso de mortalidad en noviembre de 2009, durante las semanas 46, 47 y 48 (del 9 al 29 de noviembre), lo que representó 41 defunciones observadas frente a las 20 esperadas (tabla 2).

Las 41 defunciones observadas entre cinco y 14 años se registraron en 29 municipios de los cuales $23(79,3 \%)$ tuvieron una única defunción. Los 6 municipios que presentaron más de 1 defunción siguieron el mismo patrón de mortalidad observado durante el resto del año.

\section{Gripe A(H1N1) 2009}

El Sistema de Vigilancia de la Gripe en España identificó el pico de máxima incidencia de la onda pandémica de gripe en la semana 47/2009 (del 16 al 22 de noviembre). Sin embargo, al igual que en temporadas estacionales previas de gripe, la máxima tasa de detección viral, que indica una mayor intensidad de la circualción viral, se produjo tres semanas antes, en la semana 44 (del 26 de octubre al 1 de noviembre de 2009). A partir de esta semana se inició el descenso de la onda hasta la semana 52/2009 (del 21 al 27 de diciembre), en la que se volvieron a registrar tasas de incidencia de gripe por debajo del umbral basal establecido para la temporada 2009-2010. (figura 3).

Durante el año 2009 se notificaron 286 defunciones confirmadas por virus de la gripe (H1N1) 2009 al Centro de Coordinacion de alertas y emergencias sanitarias del Ministerio de Sanidad y Política Social, responsable del seguimiento de las defunciones causadas por virus pandémico en España. Durante los dos periodos de exceso de mortalidad identificados por MoMo, semanas 46-47/2009 (466 defunciones de exceso) y semanas 51-52/2009 (483 defunciones de exceso), se notificaron un total de 49 y 33 defunciones por gripe (H1N1) 2009 respectivamente.

En el grupo de edad de 5 a 14 años durante las semanas 46-48/2009 se notificaron 5 defunciones por gripe A(H1N1) 2009.

\section{Virus Sincitial Respiratorio}

La tasa de detección de VRS aumentó rápidamente entre las semanas 47-50/2009 
Figura 3

Incidencia de gripe, Tasa de detección de gripe y tasa de detección VRS, semanas 22-53/2009

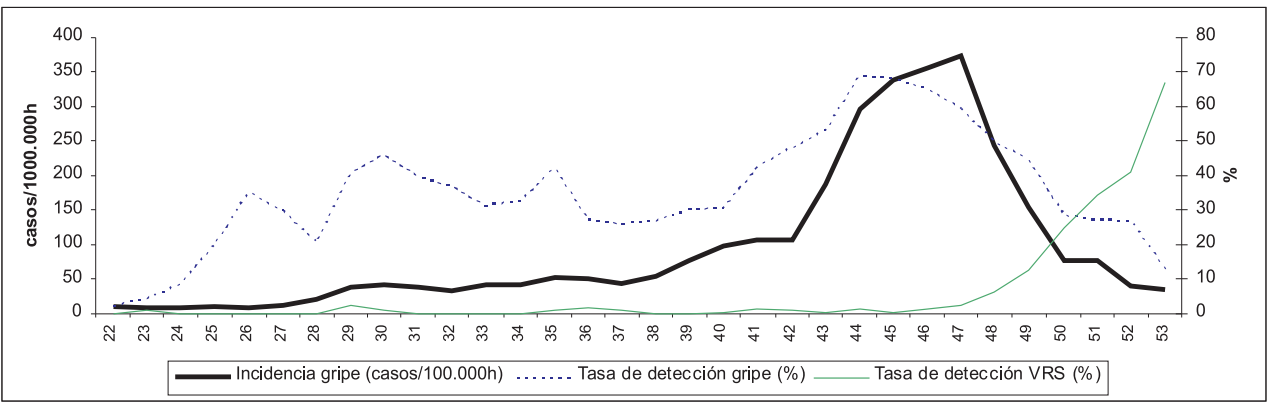

alcanzando su pico máximo en la semana 53/2009. A diferencia de temporadas estacionales previas de gripe, la circulación de VRS en la temporada pandémica 2009-2010 se presentó con posterioridad a la de virus gripales, sin el habitual solapamiento de ambos virus respiratorios en las semanas de máxima intensidad de circulación viral. (figura 3).

\section{DISCUSIÓN}

En los meses de noviembre y diciembre se observaron dos periodos de mortalidad moderadamente por encima de lo esperado. El de noviembre se inció en la semana 46/2009 y terminó en la semana 47/2009 y el de diciembre se inició en la semana 51/2009 y terminó en la semana 52/2009. Estos periodos tuvieron una duración significativamente mayor de lo esperado y la mortalidad observada superó dos días en cada periodo, el umbral de alerta para la mortalidad diaria esperada (IC95\%). En los niños de entre 5 y 14 años también se observó un periodo de mortalidad por encima de lo esperado en noviembre, entre las semanas 46-48/2009.

Tanto en la población general como en el grupo de edad de 5 a 14 años, los excesos de mortalidad de noviembre se produjeron antes o concomitantemente con la máxima incidencia de gripe.
El segundo exceso de mortalidad observado durante el mes de diciembre se produjo durante un periodo de baja intensidad de circulación de virus gripales, con tasas de incidencia por debajo del umbral basal establecido para la temporada 2009-2010 y cinco semanas después del pico máximo incidencia de gripe. De acuerdo con el Sistema de Vigilancia de la Gripe en España y el sistema MoMo, los máximos excesos de mortalidad por todas las causas se han observado en las últimas temporadas estacionales de gripe (desde la temporada 2004-2005) con un retraso de entre una y tres semanas del pico máximo de actividad gripal.

Entre las semanas 46 y 48 de 2009 se identificaron 41 defunciones por todas las causas en el grupo de edad de 5 a 14 años, de las cuales cinco defunciones estaban asociadas a la gripe (H1N1) 2009. Aunque en las defunciones en adultos la gripe podría no ser identificada como causa de muerte en algunas de ellas, en el grupo de edad de 5 a 14 años la causa de muerte es analizada exahustivamente y es difícil pensar que una defunción en este grupo por (H1N1) 2009 durante un periodo de pandemia se hubiera quedado sin identificar. Además, en el periodo de interés no se observó ningún agrupamiento espacial en ningún grupo de edad.

El inicio en el incremento de detección de VRS se produjo durante el primer periodo 
de exceso de mortalidad identificado en noviembre y su pico se alcanzó una semana después del exceso de mortalidad identificado en diciembre.

Los informes de la Agencia Estatal de Metereología ${ }^{6,7}$ indicaron que el mes de noviembre fue muy cálido en la mayor parte de España, sin embargo el mes de diciembre fue en su conjunto algo más frío de lo normal. A partir del día 13 de diciembre las temperaturas descendieron de forma notable, manteniéndose en general muy bajas hasta el día 21 , con fuertes heladas en el interior peninsular, que fueron especialmente intensas en las madrugadas de los días 16, 19 y 20, en las que las temperaturas descendieron por debajo de los $10^{\circ} \mathrm{C}$ en numerosos puntos. El exceso de mortalidad por todas las causas detectado en la semanas 51-52/2009 coincidió en el tiempo con una notable bajada de temperaturas y la publicación por parte de la AEMET de una alerta por ola de frío en los días 19 y 20 de diciembre de 2009.

El exceso detectado durante el mes de noviembre no se puede explicar por variables climáticas. Sin embargo el brusco descenso de temperatura observado desde el final de la semana 49/2009 hasta final de año podría explicar el exceso de mortalidad identificado durante el mes de diciembre.

Tras consultar los registros diarios de accidentes de tráfico en carretera con victima mortal a las 24 horas, también se ha podido descartar esta como causa de los excesos de mortalidad identificados.

Varios factores pueden haber contribuido a los excesos observados de mortalidad por todas las causas en España, durante algunas semanas de noviembre y diciembre de 2009, incluyendo factores no valorados en este estudio, como podrían ser exceso de polución, otras variables climáticas (humedad absoluta,...), procesos alergicos asociados a polen otoñal que han podido alargarse en el tiempo debido a las altas temperaturas en el caso particular de noviembre. La temprana presentación en el tiempo de la onda pandémica por (H1N1) 2009 respecto a previas ondas epidémicas estacionales de gripe no se puede descartar como uno de los factores que han contribuido al exceso de mortalidad observado en noviembre, además posibles sesgos asociados a la notificación de defunciones por gripe (H1N1) 2009 podrían subestimar el peso de este factor. Factores climáticos explicarían mejor el exceso observado en diciembre, aunque no se puede descartar una contribución del VRS a dicho exceso observado.

MoMo no incluye ni variables meteorológicas ni causa de muerte en su análisis, por lo que no permite establecer causalidad en los análisis realizados por el sistema. Para establecer causalidad en los excesos detectados es necesaria una investigación a nivel local. Pese a las limitaciones inherentes a los sistemas de vigilancia, estos resultados muestran la capacidad de MoMo para identificar de forma oportuna situaciones de riesgo para la salud pública y la importancia de la mortalidad general como indicador indirecto de diversos problemas de importancia en salud pública.

\section{BIBLIOGRAFÍA}

1. Mazick A, Gergonne B, Wuillaume F, Danis K, Vantarakis A, Uphoff H, Spiteri G, van 't Klooster T, Junker C, Holmberg M, Molbak K.Higher allcause mortality in children during autumn 2009 compared with the three previous years: pooled results from eight European countries. Euro Surveill. 2010;15(5). pii: 19480 .

2. Instituto Nacional de Estadística. Explotación estadística del censo municipal hasta el 1 enero 2007. Disponible online en: http://www.ine.es/jaxi/menu. do? type $=$ pcaxis $\&$ file $=$ pcaxis $\&$ path $=02 \mathrm{Ft} 20 \% 2 \mathrm{Fe}$ $245 \% 2 \mathrm{Fp} 05 \% 2 \mathrm{~F} \% 2 \mathrm{Fa} 2007$.

3. Hutwagner LC, Maloney EK, Bean NH, Slutsker L, Martin SM. 1997. Using laboratory-based surveillance data for prevention: an algorithm for detecting Salmonella outbreaks. Emerg Infect Dis. 3:395-400. 
4. International Standard ISO 8601: 1988 (E), «Data elements and interchange formats -Information Exchange- Representation of dates and times». Geneva :International Organization for Standardization; 1988.p.3-17.

5. Centro Nacional de Epidemiología del Instituto de Salud Carlos III. Sistema de Vigilancia de la Gripe en España. Red Nacional de Vigilancia Epidemiológica. Área de Vigilancia de la Salud Pública. Centro Nacional de Epidemiología del Instituto de Salud Carlos III. (http://vgripe.isciii.es/gripe).
6. Agencia Estatal de Meteorología. Resumen mensual del clima: noviembre 2009. Disponible en: http://www.aemet.es/documentos/es/elclima/datos _climat/resumenes_climat/mensuales/2009/res_m ens_clim_2009_11.pdf.

7. Agencia Estatal de Meteorología. Resumen mensual del clima: diciembre 2009. Disponible en: http://www.aemet.es/documentos/es/elclima/datos _climat/resumenes_climat/mensuales/2009/res_m ens clim 2009 11.pdf. 